\title{
LAS PRÁCTICAS MATEMÁTICAS DE IDENTIFICACIÓN DE PATRONES: DOCENCIA Y ARTE PRECOLOMBINO
}

\author{
MATHEMATICAL PATTERN \\ IDENTIFICATION PRACTICES: TEACHING \\ AND PRE - COLUMBIAN ART
}

\section{Jeannette Vargas Hernández ${ }^{1}$}

Nury Vargas Hernández ${ }^{2}$

Juan José Castro Cerón ${ }^{3}$

Universidad Colegio Mayor

de Cundinamarca

$1 \quad$ PhD Educación Matemática. Universidad de Salamanca España. ORCID https://orcid.org/0000-0002-8936-696X Universidad de Salamanca España. jeannettevargash@usal.es 2 Maestra en Artes Plásticas. Universidad Nacional de Colombia. Estudiante en Magister en Estética e Historia del Arte. Universidad Jorge Tadeo Lozano. Bogotá ORCID https://orcid.org/0000-0002-8210-6719 nvargash@unicolmayor.edu.co 3 Maestría en Análisis y Visualización de datos masivos BigData. Universidad Internacional de la Rioja. España. ORCID https://orcid.org/0000-0002-4698-7096 Universidad Colegio Mayor de Cundinamarca jjosecastro@unicolmayor.edu.co

\section{RESUMEN}

La presente propuesta de innovación se deriva de una indagación transdisciplinaria, descriptiva y cualitativa que sustenta el proyecto de aula, el cual se realiza para propiciar el aprendizaje basado en la teoría de la práctica social. La innovación en el primer semestre universitario requirió del apoyo al docente por parte de los investigadores desde las artes plásticas y la educación matemática. El acento está en la experiencia de participación de estudiantes y profesores universitarios, en un intento de permitir una construcción colectiva alrededor de las prácticas matemáticas de identificación y uso de regularidades y patrones, mediadas 
por la observación, creación y razonamientos inductivos alrededor de la iconografía precolombina. Se presenta una descripción de la empresa en común concerniente a creación de regularidades, uso de patrones, de escalas, creaciones en 3D y estructuración de razonamientos inductivos con un seguimiento desde la participación que se observa en la comunidad de práctica de la clase.

\section{PALABRAS CLAVE}

regularidades y patrones, figuras precolombinas, profesores universitarios, artes plásticas, prácticas matemáticas.

\section{ABSTRACT}

The present innovation proposal is derived from a transdisciplinary, descriptive, and qualitative inquiry that supports the classroom project, which is carried out to promote learning based on the theory of social practice. The innovation, in the first university semester, requires the support of the teacher by the researchers, from the plastic arts and mathematics education. The purpose is on the participation experience of university students and professors, in an attempt to allow a collective construction around the mathematical practices of identification, and use of regularities and patterns, mediated by observation, creation, and inductive reasoning around pre-Columbian iconography. A description of the common enterprise is presented regarding the creation of regularities, the use of patterns, scales, $3 \mathrm{D}$ creations, and the structuring of inductive reasoning, with a follow-up from the participation that is observed in the community of practice of the class.

\section{KEYWORDS}

regularities and patterns, pre-Columbian figures, university professors, plastic arts, mathematical practice

\section{INTRODUCCIÓN}

El mundo contemporáneo nos invita a replantear la formación académica que hemos venido llevando por muchos años y de allí, la manera de actuar del docente, para que a través de su trabajo contribuya en la formación de personas que tengan la capacidad de solucionar problemas, con habilidades comunicativas y por supuesto la habilidad de identificación y sistematización de la información.

Los ambientes de aprendizaje, acompañados de deliberación y puesta en marcha de propuestas de enseñanza, en el caso que nos interesa "la práctica matemática de identificación y uso de regularidades y patrones" desde la perspectiva de la educación matemática y en interdisciplinariedad con las artes plásticas, se convierten en espacios de reflexiones y acciones, en los cuales se propicia el estudio de referentes teóricos y uso de perspectivas como en el caso, de las comunidades de práctica del aula. Ambientes que permitan traer los resultados de la investigación al aula y requieren del planteamiento de preguntas por parte del profesor; esbozo de problemas que trasciendan y alimenten la docencia con investigación.

Steen (1988) y diversos autores se refieren a los patrones matemáticos como la esencia de las matemáticas. Aquí los interpretamos, similar a Morales, Cañadas y Castro (2017), como una estructura que permite modelizar las reiteraciones que se observan en el entorno y mediados por la abstracción se convierten en objetos del pensamiento.

Por otro lado, siguiendo a Read (1990), se asume la noción de arte como otra forma de conocer, cercana al conocimiento desde la ciencia o la filosofía, considerando el arte, como parte fundamental para el desarrollo del individuo en cualquier etapa. Así, el acercamiento al arte precolombino, enfocado en el estudio de patrones y regularidades en 
figuras y objetos específicos, se realiza desde el manejo de una serie de conceptos y elementos que, considerando a Sondereguer (2003), se evidencian en el estudio de las representaciones plásticas y el diseño en análisis de diferentes cánones morfoproporcionales, Respecto a los elementos comunes en la iconografía es reiterativo identificarlos en representaciones de felinos, serpientes y aves, especialmente.

El arte precolombino se caracteriza por su alto contenido simbólico, y al respecto, dentro del tema de interés del actual planteamiento, se consideran los aportes de Reichel-Dolmatof (2005), como antropólogo, desde su disciplina realizó investigación etnográfica, dando cuenta de un entendimiento y acercamiento que nutre la contextualización de las figuras precolombinas. Al proponer un análisis del tema de patrones y regularidades a partir de objetos orfebres precolombinos y centrar la atención en la iconografía, se considera a Sondereguer (2000), en cuanto a la definición puntual de lo iconográfico como "el método analíticodescriptivo vinculado al aspecto antropológico e histórico y relacionado con el entorno cultural de la obra visual plástica"(p. 39). Se tiene así que lo iconográfico va dirigido a una descripción morfológica, en cuanto que lo iconológico gira hacia una interpretación morfológica, "se refiere al método analítico- interpretativo de los aspectos simbólico y expresivo, metafísico, semiológico y estético de la obra visual plástica" (p. 39). Desde otra posibilidad de estudio, Legast (1987), proporciona un reconocimiento de las especies representadas, siendo analizadas e identificadas desde la biología. Tanto el reconocimiento de iconos como el de especies animales, es clave en la comprensión y elección de las figuras susceptibles a ser analizadas bajo el estudio de las regularidades y patrones o elementos característicos como se explicará en el texto.
A partir del estudio de Rojas (2013), se encuentra un recurso para explorar en las reseñas de algunas investigaciones relacionadas con la Etnomatemática y especialmente se ubica en la caracterización de Blanco (2006), en lo referente a la utilización de elementos y objetos de arte precolombino como herramientas pedagógicas en la enseñanza de la matemática.

Aunado a lo anterior, en este proyecto de aprendizaje en el cual se enfatiza en aprender participando y aprender trabajando con los demás, se retoman las nociones de comunidades de práctica. En este sentido, a partir de Wenger (1998) se tiene en cuenta que hay tres características propias de una comunidad de práctica, que son:

Dominio: un interés común que conecta y se mantiene unido a la comunidad.

Comunidad: una colectividad comprometida a través de actividades compartidas que persiguen un dominio común.

Práctica: los miembros de una comunidad de práctica son practicantes; lo que hacen involucra su participación en la comunidad; y lo que aprenden de la comunidad afecta a lo que hacen.

Para asociar práctica y comunidad hay que describir tres dimensiones que alimentan a una comunidad, abordando la teoría de Wegner (1998) estas son: el compromiso mutuo, la empresa común y el repertorio compartido. La primera es la esencia de una comunidad de práctica, ya que las relaciones de participación mutua y la acción de cada uno de sus integrantes le va dando significado a una comunidad, es decir la comunidad de práctica no puede ser un sinónimo de grupo. 
Figura 1. Dimensiones de la práctica como propiedad de una comunidad. Diagrama a partir de Wenger (1998, p. 100)

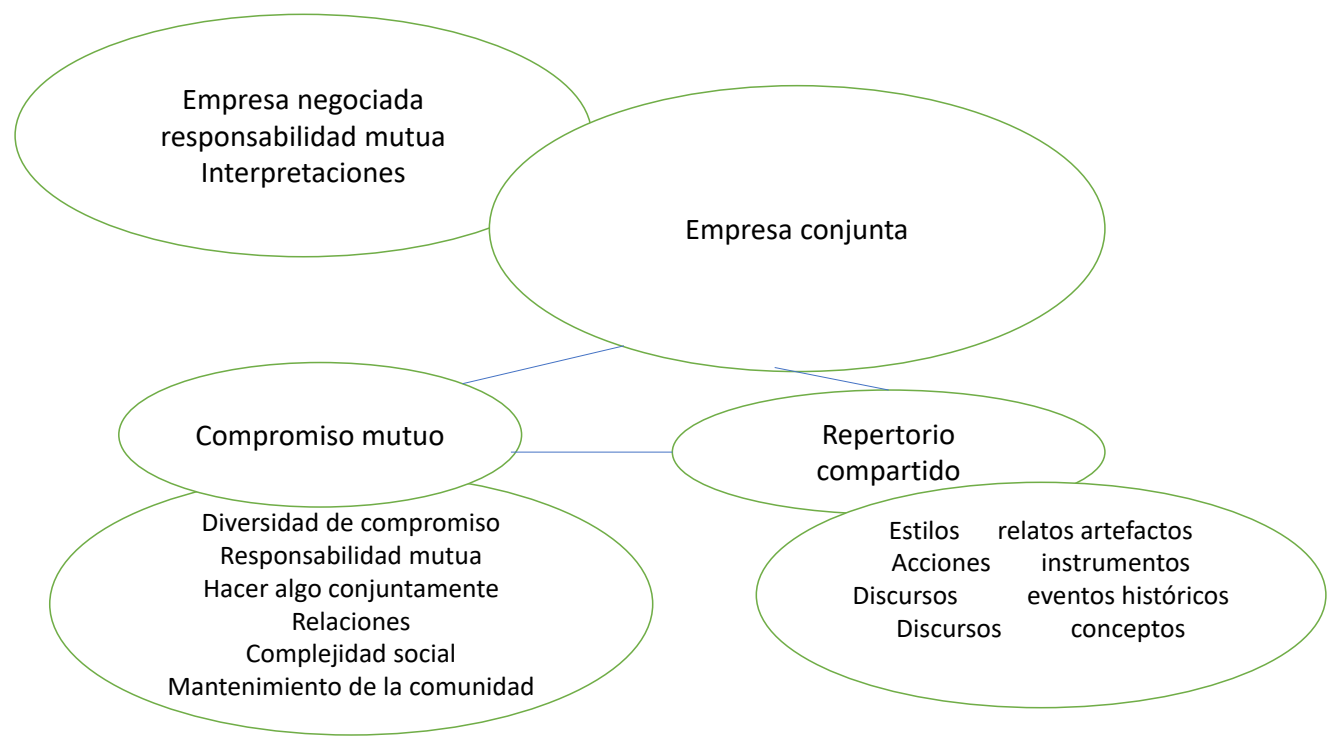

En lo que refiere a la empresa común, es la negociación de una empresa conjunta, es el resultado de un proceso colectivo, ésta la definen los participantes convirtiéndose en una parte integral de la práctica. Si el compromiso mutuo no exige homogeneidad, la empresa común no supone un acuerdo simple sino una negociación colectiva y autónoma.

En cuanto a la tercera característica el repertorio compartido de una comunidad de práctica incluye repertorios, palabras, manera de hacer gestos o símbolos y estilos de expresión, entre otros, que se han adoptado al interior de ella en el curso de su existencia y que han pasado a formar parte de su práctica y le dan a la comunidad una identidad propia (Figura 1).

Si bien, el argumento es que los individuos aprenden a través de la participación en una comunidad de práctica y se espera la generación de nuevos niveles de conocimiento, mediante la actividad al interior del grupo. En esta investigación se emplea el término acuñado por
Clark (2005), 'comunidad de práctica de clase' y se caracteriza acudiendo a Camargo 2010 así:

'Una 'comunidad de práctica de clase' 192 es una configuración social en donde el profesor y los estudiantes llevan a cabo una empresa de su interés. A través de la participación y el deseo de sacar adelante la empresa ocurre el aprendizaje de los estudiantes como un efecto de la práctica. Aunque el objetivo principal de los estudiantes es aprender y el del profesor es lograr que sus estudiantes aprendan, es posible proponer a los estudiantes la consecución de una empresa conjunta, diferente a la de aprender, y organizar la clase en función de ésta, buscando con ello hacer partícipes a los estudiantes de la definición de dicha empresa. En ese sentido, aunque los estudiantes no se agrupan inicialmente motivados por contribuir en una empresa que se les propone, es posible que terminan involucrándose en sacarla adelante" ( $p$. 61). 
En su caracterización la autora arriba citada, expone que en una comunidad de práctica de clase los estudiantes tienen la posibilidad de moverse hacia la posición de expertos contando con las relaciones democráticas que intenta establecer el profesor. Éste puede aprovechar la relación asimétrica que sostiene con sus estudiantes para disminuir el efecto limitante de la estructura de poder en una clase -en donde el profesor tiene la autoridad de la calificación y la participación de los estudiantes puede estar motivada por temor a obtener malas calificaciones y no por un interés genuino (Camargo, 2010).

Lave y Wenger (1991) usan la expresión 'participación periférica legítima' para referirse al proceso -característico del aprendizajemediante el cual los recién llegados a una comunidad se integran a esta. En el ámbito educativo, atendiendo a Camargo (2010), al considerar una clase como una comunidad de práctica, se puede usar la expresión "participación periférica legítima" para hacer referencia a la evolución en la participación personal de los estudiantes a medida en que avanzan colectivamente en las actividades matemáticas propuestas.

\section{MÉTODO}

El trabajo que se presenta se fundamenta en una investigación en educación matemática, que genera una propuesta transdisciplinar a manera de invitación dirigida a los profesores para incursionar en las nociones de las comunidades de práctica. Esta iniciativa busca dotar a los docentes con una idea flexible de intervención en el aula, la cual exige que ellos estudien, comprendan y se identifiquen con estas nociones.

Se organiza en varias facetas: la de planeación, seguida de la formación y organización del equipo que implementa la propuesta y luego la implementación junto con el análisis de sus resultados.

La propuesta tiene una faceta de planeación, con una duración de un año y contó con expertos en las áreas de artes plásticas, investigación en educación matemática y docencia. Se genera por el interés en indagar sobre ¿Cómo propiciar en estudiantes de primer semestre universitario las prácticas de identificación y uso de regularidades y patrones, desde las artes plásticas y la didáctica de la matemática?

En el ámbito de la Educación Matemática y con una mirada distinta a la tradicional, se determina el contexto artístico como un espacio que privilegia un trabajo interdisciplinario entre profesores, con el propósito de presentar un enfoque en la enseñanza en los primeros cursos universitarios de precálculo. Como temática transversal en estos estudios se reconocen las prácticas matemáticas de identificación y uso de patrones, y desde las artes plásticas y visuales, se examinan las culturas y zonas arqueológicas precolombinas Muisca y Tairona. Se caracterizaron figuras de estas regiones tanto por su simbología como por su posibilidad de ser analizadas desde semejanzas y diferencias formales, por ejemplo, hacer precisiones en cuanto a las medidas, diseños geométricos o esquemáticos, entre otros.

Se estableció una propuesta de trabajo en el aula, que se formaliza a través de diversas tareas en un taller guía, el cual se presenta detalladamente, para los profesores de Precálculo ${ }^{4}$ del primer semestre universitario; determinaciones fundamentadas a partir de una profunda reflexión y estudio documental (Vargas, Vargas y García, 2020).

Para la implementación se presenta un esbozo de los planteamientos que el profesor tendrá

\footnotetext{
$4 \quad$ La asignatura de Precálculo en Colombia designa
} a los cursos anteriores al estudio del análisis matemático. 
como guía en su gestión en el aula y que, podrá refrendar con su grupo de estudiantes:

"EMPRESA COMÚN: Identificación y uso de regularidades y patrones, concretamente en figuras precolombinas y el descubrimiento y uso de esta práctica en el aprendizaje de otros conceptos matemáticos.

COMPROMISO MUTUO: Participar en los talleres y discusiones concernientes a regularidades y patrones. Generar propuestas de identificación y uso de patrones en figuras precolombinas de diferentes zonas arqueológicas, así como en conceptos matemáticos.

\section{REPERTORIO COMPARTIDO:}

Estrategias de acercamiento a las regiones arqueológicas denominadas precolombinas, contextualización de lo precolombino como legado cultural, rutinas en la selección y el examen de imágenes, uso de vocabulario, desde las artes plásticas, y su tránsito a estrategias de acercamiento al uso de regularidades y patrones en lo relativo a conceptos matemáticos" (Vargas, Vargas y García, 2020, p.325).

La caracterización de una comunidad de práctica es incongruente con formatos rígidos y prescripciones establecidas previamente, dado que en el seno de las comunidades de práctica en el aula, se van generando los procesos de participación con los que se van delineando los pasos posteriores, por ello las propuesta de innovación para las prácticas matemática de identificación de regularidades y patrones, es solo un bosquejo en el cual tanto expertos como novatos intervendrán conformando los compromisos y el repertorio a través de tres sesiones de clase de 90 minutos cada una, así:
-Sesión Uno: Un primer momento que es la Conferencia de presentación y análisis de elementos característicos de un icono precolombino, con el soporte del vídeo "Regularidades y patrones, imagen del Hombre -Murciélago en la cultura Tairona", diseñado para tal fin (https://www.youtube.com/ watch?v=6uQ98wJoJdg). El segundo y tercer momento, respectivamente, van dirigidos a la guía y análisis por parte del profesor y a las reflexiones de los estudiantes respecto al tema regularidades y patrones.

El material visual titulado Regularidades $y$ Patrones. Imagen del Hombre-murciélago en la cultura Tairona, contiene las reflexiones, búsqueda y estudio de información teórica y visual, observación y análisis del tema de regularidades y patrones 0 elementos característicos en un grupo específico y reducido de objetos orfebres, con dedicación a un icono específico de la cultura y zona arqueológica precolombina Tairona, en Colombia.

Por eso, se presenta primero la contextualización del estudio previo de la imagen precolombina, a continuación, está el momento de la recopilación y selección de las figuras para analizar, luego se identifica la fase de planteamiento y definición de su utilización, continuando con la terminología específica y la elaboración de material visual necesario dentro de la estructura de la propuesta, de donde surgen las carpetas de trabajo y el vídeo que se implementa como soporte de la Sesión 1.

Respecto a la contextualización, desde las artes plásticas y visuales, se observa la producción artística en zonas arqueológicas específicas de América como un tema pertinente para abordarlo en talleres experimentales de arte con los grupos de estudiantes universitarios, al tiempo que permite estructurar posibilidades que son parte fundamental en el trabajo interdisciplinario 
que realiza el grupo de investigación (Vargas y Vargas, 2019).

La recopilación y selección de las figuras precolombinas se realiza desde el archivo particular de una de las investigadoras; con la intención que los estudiantes puedan observar, con nuevos ojos, los objetos y sus detalles, leer información visual y teórica, tomar nuevas fotografías y ampliar el archivo.

Actividad previa a la sesión dos: visita al Museo del Oro.

\section{- Sesión Dos: Posterior a visita al Museo del Oro.}

Trabajo de observación y estudio del material visual dispuesto en carpetas para ser explorado en grupos de estudiantes. Los grupos de estudiantes determinarán la estrategia y uso del material informativo suministrado en los formatos del taller, estructurados para servir de guía en la identificación de las culturas y zonas arqueológicas, tanto Muisca como Tairona.

Los grupos de estudiantes intentarán llegar a un consenso sobre lo que van a proponer; los procesos de negociación de significados y la participación al interior del grupo. El proceso de generación de estrategias y examen de regularidades y patrones de repetición, serán las manifestaciones con las que cuenta el profesor para el seguimiento del aprendizaje.

- Sesión Tres: por grupos de trabajo, los estudiantes esbozan y crean un nuevo diseño o figura que surge desde el examen de las imágenes proporcionadas en las carpetas, para luego sustentarlo desde el tema Regularidades y Patrones, así como desde la figura precolombina y el arte, según la indagación y entendimiento al cual el grupo ha llegado.

Esta propuesta incluye una rúbrica de evaluación que establece tres niveles de participación y adicionalmente se recurre a categorías con descriptores para establecer aspectos de dicha participación, tales como: demanda de ayuda del estudiante al profesor; utilización de recursos para la actividad, fundamentación de las ideas, relevancia de las ideas, autonomía y originalidad. 
Tabla 1. Rúbrica niveles de participación de los estudiantes

Fuente: Vargas, Vargas y Cáceres 2020.

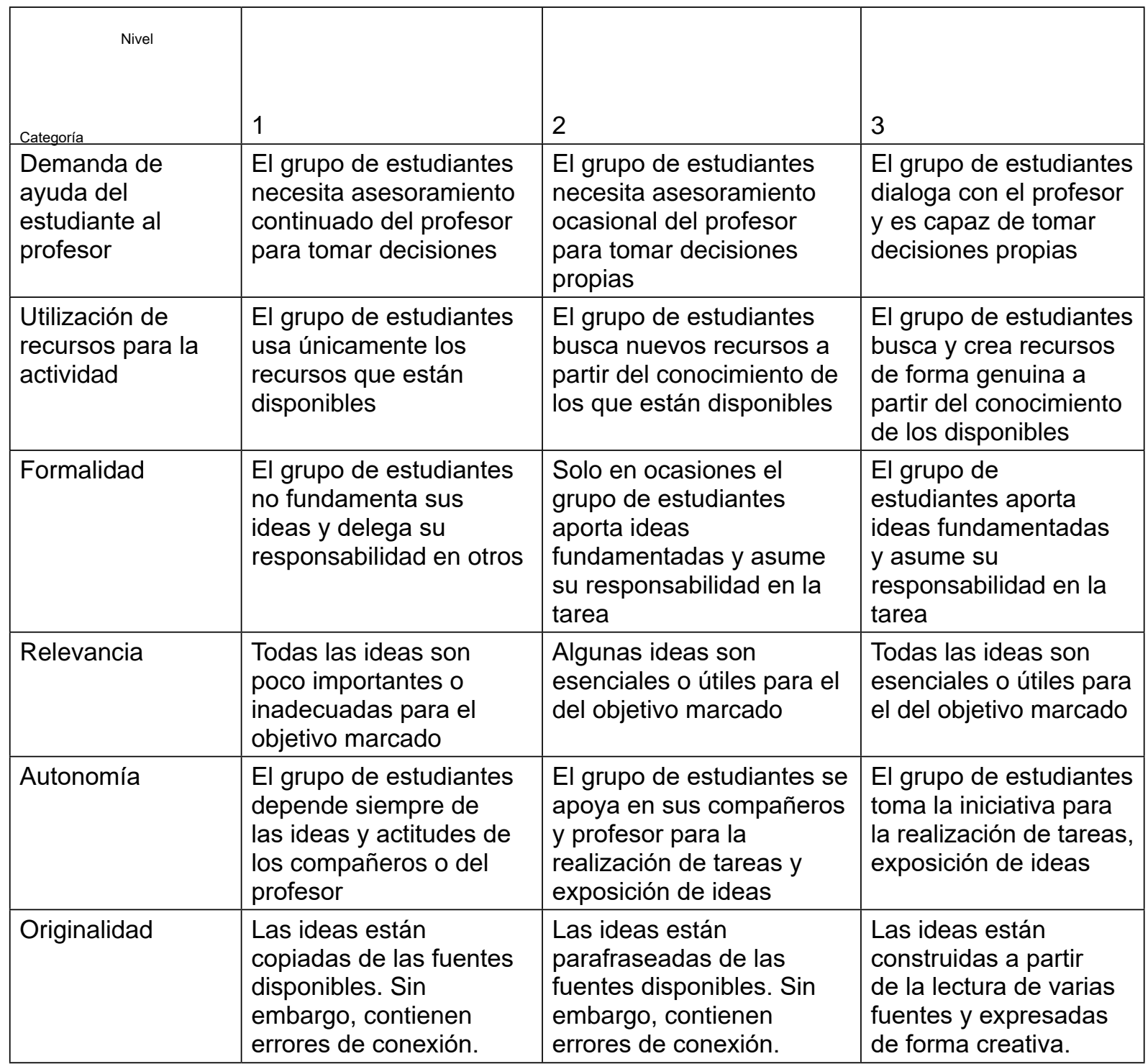

A partir del análisis de datos en la rúbrica, se considera Participación periférica legítima si la valoración media de los aspectos se encuentra en el intervalo [1,2); Participación legítima si la valoración media de los aspectos se encuentra en el intervalo $[2,3)$ y Participación plena si la valoración media de los aspectos es 3 .

\section{FASE DE FORMACIÓN Y ORGANIZACIÓN DEL EQUIPO QUE IMPLEMENTA LA INNOVACIÓN}

Se suma al equipo de investigadores un docente de matemáticas quien no conoce la propuesta y será uno de los encargados de llevarla a término. El docente encargado e interesado en la puesta en práctica de la propuesta de innovación, hace estudio de ella (Vargas, Vargas y Cáceres, 2020). 
Se llevan a término dos sesiones entre los investigadores y el docente. Una de ellas para ver y analizar el video que mediará en el repertorio compartido. En la segunda sesión entre investigadores y docente, se escuchan las inquietudes del docente y se conversa sobre la forma en la cual se creó la innovación, sus objetivos y las sesiones del taller.

Se hacen adecuaciones a la propuesta original y se genera en esta innovación la posibilidad de invitar a los estudiantes en dos oportunidades a la visita al Museo del Oro. Se acuerda que se llevarán a término entregas de los grupos, correspondientes a los trabajos parciales y finales y también se realizarán socializaciones de las creaciones que realicen los grupos. Además, se plantea la necesidad de una sesión final a la cual se le denomina de Consolidación.

En cuanto al desarrollo de las sesiones se acuerda que siempre que sea posible, el profesor en las socializaciones puede designar a un estudiante que controla el tiempo para cada grupo y él se limita a ceder la palabra a los estudiantes y preguntar al grupo cuando alguna parte de la información está errada. El profesor es quien siempre cierra la sesión y propende porque en la socialización se llegue a conclusiones referidas a qué se entiende por patrones y regularidades y el tipo de patrones utilizados.

\section{FASE DE IMPLEMENTACIÓN}

En la implementación de esta innovación participa un equipo integrado por dos de las investigadoras; profesoras de matemáticas y de artes plásticas respectivamente y un docente profesor con amplia experiencia en cursos universitarios de Precálculo y Cálculo. El profesor de matemáticas enseñaba a 23 estudiantes de primer semestre en el programa de pregrado de Laboratorio Clínico y Bacteriología en Bogotá, Colombia.

\section{Sesión 1: Conferencia.}

\section{Presentación y análisis de elementos característicos en la identificación del hombre-murciélago; icono precolombino en la cultura y zona arqueológica tairona.}

Para el momento de la conferencia, por solicitud realizada con antelación, los estudiantes, ya han revisado el vídeo "Regularidades y patrones, imagen del hombre-murciélago en la cultura tairona" elaborado para brindar soporte visual y teórico del tema propuesto.

La sesión se enfoca en ampliar y detallar la reflexión concerniente a regularidades y patrones lo que, desde la respectiva investigación, se propone llamar "elementos característicos" del icono denominado por la antropología, hombremurciélago; correspondiente a la cultura y zona arqueológica Tairona.

En la conferencia se realiza la presentación de extractos específicos del vídeo ya mencionado, con el objetivo de puntualizar en la información y análisis pertinente de aspectos relacionados con los "elementos característicos" que hacen reconocible e identificable la imagen del icono, evidenciando su conexión con otras representaciones que, en la misma región arqueológica son susceptibles de ser estudiadas, continuando la metodología propuesta en el análisis del icono del hombre-murciélago. Dentro de la sesión se presenta una síntesis que brinda a los estudiantes la ubicación geográfica, temporal y contextualización del grupo y zona precolombina. También se enfatiza el hecho de que el video se sustenta en investigaciones que, en Colombia, han sido realizadas especialmente por antropólogos y biólogos alrededor de objetos orfebres y cerámicos, los que en su mayoría se encuentran en el Museo del Oro del Banco de la República. 
Con la proyección de un mapa de Colombia, que en algunas ocasiones no es tan conocido por los estudiantes como se esperaría, se inicia una interacción de reconocimiento de un territorio, para después, con la imagen del hombre murciélago tairona o el tunjo muisca con todos sus elementos característicos y terminología específica, acompañar a los estudiantes a involucrarse y conectarse con el tema artístico, cultural y simbólico precolombino.

Se pretende que surja una conversación con los estudiantes y también con el profesor de matemática, quien está presente durante la conferencia y quien efectivamente está conectado con la actividad realizando intervenciones que vinculan aún más a los estudiantes con el tema desde la matemática y hacia próximos trabajos que realizarán con base en el enfoque de la conferencia.

\section{Sesión 2 de implementación. Razonamiento inductivo y creación de nuevos patrones de repetición.}

Posterior a la inmersión del análisis del video en compañía de la maestra de artes plásticas, los estudiantes son invitados a realizar una visita al Museo del Oro.

Para la clase con el profesor de matemáticas, los estudiantes traen un sinnúmero de fotografías, resultado de la visita al Museo del Oro, que utilizaran para enriquecer alguna de las carpetas con fotografías clasificadas que se les facilitan en medio magnético o a través de la plataforma.

Inicialmente los estudiantes revisaron dos de las cinco diferentes carpetas, diseñadas para este trabajo por la maestra de artes plásticas, tales como: Carpeta 1 (serpiente), Carpeta 2 (murciélago), Carpeta 3 (hombres - ave), Carpeta 4 (narigueras), Carpeta 5 (tunjos).
Con el material fotográfico facilitado y el recogido en el recorrido al museo del oro, el profesor solicita integrar los grupos de trabajo. Así los estudiantes se asocian libremente con lo cual se busca que el aprendizaje experiencial y las relaciones sociales se pueden combinar e integrar, a través de la observación, estudio y también debate en la búsqueda de regularidades y patrones en las fotografías de las figuras precolombinas. Adicionalmente se abre espacio a las diversas decisiones en los grupos de ir buscando información de las regiones y ubicación geográfica de ellas, de una manera similar al discurso que escucharon en la sesión anterior por parte de la maestra de artes plásticas.

Una vez el profesor considera que la organización de los grupos ha generado un compromiso por la indagación sobre vocabulario específico y reconocimiento de patrones, realiza dos propuestas de tareas, tratando de establecer un compromiso hacia la tarea que ha emprendido un grupo, la cual consiste en escribir argumentos inductivos a partir de los patrones $y$ las regularidades que han encontrado en las observaciones y en las lecturas.

La primera tarea es tomar el listado de afirmaciones con razonamientos inductivos que presentó la maestra de artes plásticas, y escribir al menos 4 afirmaciones con razonamientos inductivos. El parámetro principal que tiene el profesor en estos razonamientos inductivos es que los estudiantes examinen que ellos se caracterizan porque no afirman que la conclusión se deriva necesariamente de las premisas, sino que, de la verdad de estas, real o aceptada, es razonable inferir que, en alguna medida, la conclusión es verdadera. Si el soporte que las premisas le dan a la conclusión la hace estar más cerca de ser verdadera que de ser falsa, el razonamiento inductivo es fuerte. Pero si el soporte que le dan las premisas a la conclusión es pobre y poco sólido y la hace estar más 
cerca de ser falsa que de ser verdadera, el razonamiento inductivo es débil.

En la segunda tarea además de utilizar las conversaciones de los estudiantes, sobre las regularidades que observan en las fotografías seleccionadas en carpetas, por la profesora de artes plásticas; figuras orfebres como son los tunjos, las serpientes, hombre ave y hombre murciélago, se propone también creaciones propias. Dado que algunos estudiantes han iniciado procesos de dibujo de las figuras que están examinando, se utiliza la socialización de las indagaciones para imprimir otro nivel de exigencia, en el cual los estudiantes a través de caracterizaciones de figuras creen nuevos patrones de repetición para, posteriormente o de forma simultánea vayan realizando el dibujo de nuevas figuras precolombinas.

\section{Sesión 3. Dibujos a escala y figuras 3D}

En esta tercera sesión el profesor nota como los estudiantes se ingenian con mucha creatividad nuevas figuras a partir de las características iniciales, intentando introducirse en la mentalidad o por lo menos en la formalidad creativa de la cultura y región Muisca y de la Tairona. Estos nuevos diseños se elaboran en papel escala o cuadriculado, las figuras como se evidencia a continuación se maneja la escala ampliada y escala reducida (figura 2).

Figura 2. Muestras a escala por grupos de estudiantes.

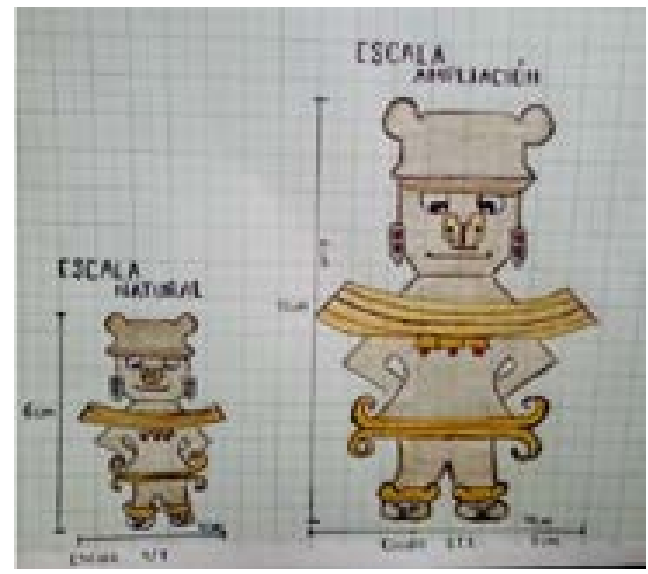

El profesor observa, que para las estudiantes este contenido se torna interesante. Realizan dibujos que denominan a escala natural de 6 $\mathrm{cm} \times 4 \mathrm{~cm}$, amplían el dibujo a la escala $2: 1 \mathrm{se}$ obtiene un dibujo de $12 \mathrm{~cm} \times 8 \mathrm{~cm}$. Otros dibujos son reducidos a la escala 1:2; en el ejemplo se tiene el dibujo original de dimensiones $10 \mathrm{~cm} \times$ $7 \mathrm{~cm}$.

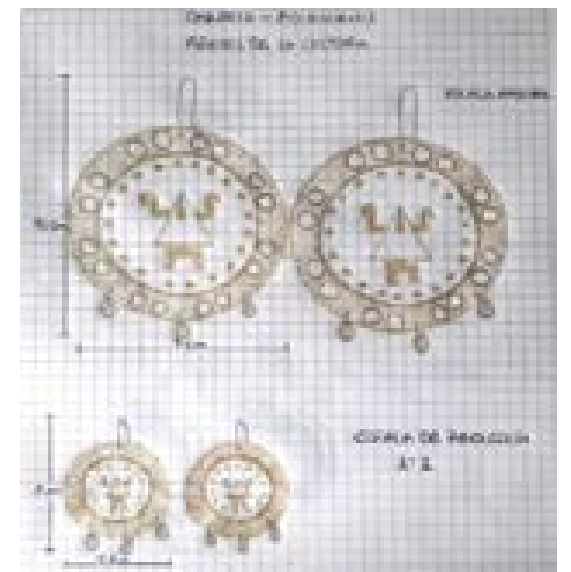

Posteriormente se distribuyen roles para elaborar las figuras a escala en 3D (Ver figura 3). 
Figura 3. Creaciones figuras precolombinas en 3D - Ampliaciones y diseños por planos.

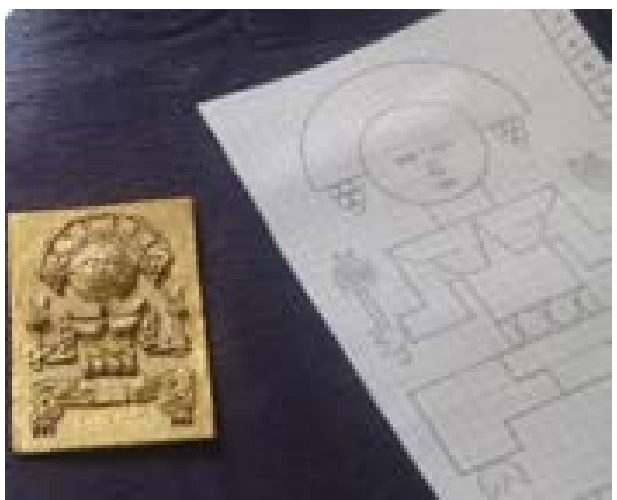

Cabe resaltar que estas figuras creadas a escala fueron elaboradas en 3D usando diferentes materiales como: arcilla, cartón y yeso. Crearon figuras detalladas asociando las indagaciones sobre las culturas o zonas arqueológicas precolombinas con nuevas representaciones que encontraron significativas y sobre todo resaltando las diferentes regularidades en busca de patrones.

\section{Sesión 4. Consolidación}

El momento que el profesor denomina taller final, que es un espacio de cierre y consolidación del trabajo realizado en el semestre, el profesor reúne trabajos finales de los estudiantes para unirlos a los trabajos parciales realizados por la comunidad de clase. Con este material se monta una exhibición y los estudiantes preparan la sustentación oral de sus creaciones.

\section{RESULTADOS DE LA IMPLEMENTACIÓN Y SU ANÁLISIS}

La información de las experiencias, en cada una de las sesiones, es recopilada por el profesor a manera de grabación o a través de los trabajos presentados por los grupos de estudiantes. Se expondrá aquí la participación a través de la producción escrita y socializaciones orales de 3 grupos que llamaremos 1B, 2D y 4E. Para la

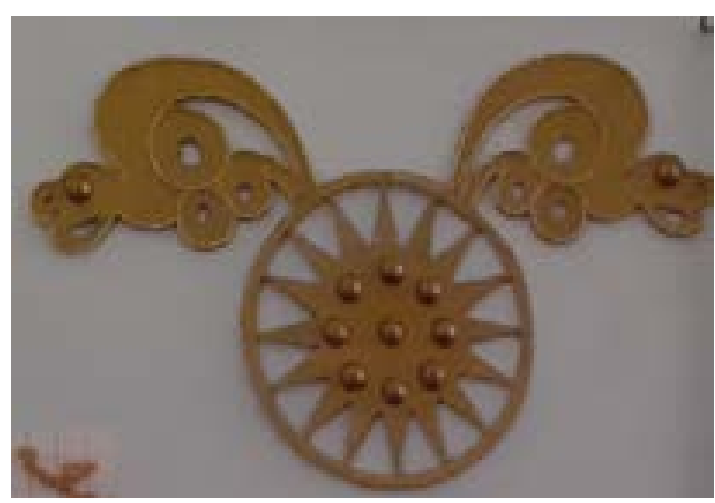

presentación se ha subdividido en las cuatro sesiones:

\section{RAZONAMIENTO INDUCTIVO Y CREACIÓN DE REGULARIDADES Y PATRONES}

\section{A. Razonamiento inductivo}

La actividad que se torna más exigente para los estudiantes consiste en la participación a través de razonamientos inductivos. De tal manera que, en el primer taller, en la producción escrita que los grupos realizan, solamente uno de ellos emprende la tarea de seguir la huella a las frases utilizadas por la maestra de artes plásticas en la exposición y redactar frases para expresar razonamientos inductivos.

Un ejemplo de la participación, (del grupo 4E), en producción escrita es:

"La figura precolombina examinada tiene forma de serpiente.

Esta figura tiene una nariz respingada.

La lengua de esta figura es bífida.

Esta figura carece de colmillos. 
Entonces la figura es probablemente una representación de la serpiente en la cultura muisca" (Grupo 4E).

Los otros grupos, emprenden esta tarea, como sugerencia dada por el profesor, en la segunda sesión de taller. A partir de lo anterior, el profesor escucha y encuentra en los escritos, en la mayoría de los casos, proposiciones "aisladas" que no permiten una probabilidad de certeza de las conclusiones expresadas.

Algunos de los razonamientos inductivos contienen solamente una sucesión de frases, sin conjeturas; con información descriptiva concerniente a características de la figura precolombina. Sin embargo, si bien en las sesiones iniciales se presenta dicha dificultad, es necesario anotar que al final del semestre, los otros dos grupos logran establecer por escrito al menos un conjunto de proposiciones de las cuales se deriva la conclusión que ellos plantean.

\section{B. Acercamiento a la identificación y uso de regularidades y patrones con conocimiento del arte y la cultura precolombina}

El profesor en una parte de las sesiones ha institucionalizado un espacio en el cual se socializa el trabajo de cada grupo de estudiantes, actividad a la cual los estudiantes han convenido acudir y en ella utilizar diversas estrategias de presentación.

Concerniente a estas socializaciones, dos de los tres grupos, por iniciativa de algunos de sus integrantes, realizan consulta sobre el significado de regularidad y patrón $y$ exponen sobre algunas regularidades en las figuras precolombinas seleccionadas por ellos. En cuanto a la participación en cada exposición, lo más destacable es que todos los estudiantes participan; grupo tras grupo expone sus imágenes, observaciones sobre ellas y otros aspectos concernientes a la cultura precolombina que eligieron, apropiándose de vocabulario especializado.

En la primera entrega, la cual se realiza a manera de exposición, con presentación en formato PowerPoint, se observa en todos los grupos un gran interés por la simbología, el cual se mantiene en todas las sesiones y el nivel de especialización en la consulta deja ver más profundidad y apropiación de los detalles que los estudiantes quieren compartir en su grupo y con los otros grupos; uno de ellos, realiza autónomamente consultas sobre mitos de la Cultura Muisca, tales como Mito de Bachué; de Chiminigagua; los Caciques de Sogamoso y Ramiriquí.

Por otro lado, en la misma línea en que la maestra de artes plásticas habló de la orfebrería, uno de los grupos consulta lo relativo a las matrices y moldes que usaban los Muiscas y el proceso de fundición en cera de abejas.

Algunos estudiantes, de dos grupos, elaboran un glosario similar al que usó la profesora de artes plásticas en la exposición inicial, y buscan un número importante de significados y vocabulario específico para integrarlo a sus explicaciones.

Dado que los estudiantes por iniciativa han consultado ejemplos de regularidades, ellos integran a estos ejemplos el caso de la escritura de la numeración en base diez. Es relevante observar que en su discurso no dan una explicación descriptiva de cuáles son las regularidades o los patrones que se establecen en este sistema de escritura.

La necesidad de trabajar con escalas, cuyo desarrollo se lleva a término en la siguiente sesión, se presenta en el momento de realizar 
la creación de una figura precolombina que conserve algunas regularidades establecidas por la cultura o región Muisca o Tairona e integrar otras o cambiar el patrón. Esta tarea, propuesta por el profesor y diseñada al interior de los grupos, en un tiempo de 30 minutos, devela algunas inquietudes de los grupos. Un ejemplo trabajado por un grupo de estudiantes, en la figura del grupo 1B (figura 4), es el siguiente:

Figura 4. Serpiente bífida creación propia

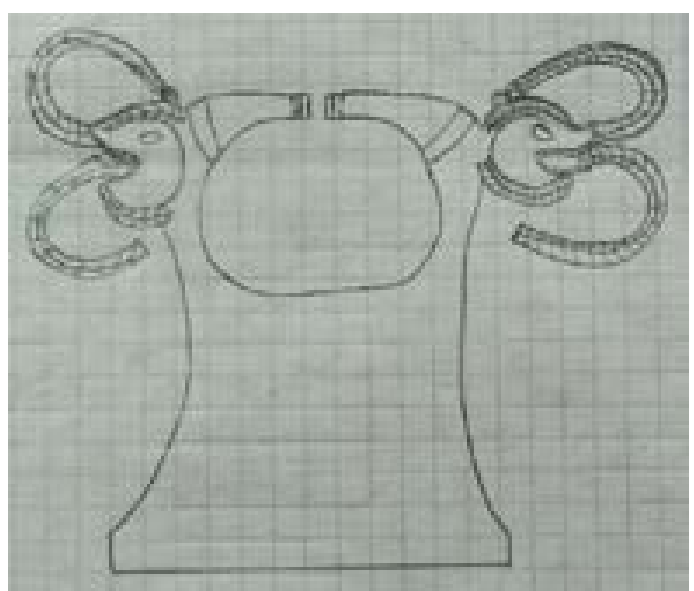

La explicación que el grupo presenta en su trabajo identifica el patrón en la cultura como el uso de serpiente con la lengua bifurcada. Enseguida escriben regularidades, tales como que el ojo de la serpiente presenta una pequeña línea vertical de lo cual se deduce que es venenosa.
La transformación consiste en que su cuerpo no está conformado por serie de círculos sino por una sucesión de líneas.

Otros grupos participan con los siguientes dibujos, en los cuales a partir de los iconos de las culturas precolombinas que estudiaron, crearon sus nuevos diseños precolombinos (figura 5).

Figura 5. Innovación figuras precolombinas

Fuente: Vargas y Vargas (2019).

Icono orfebre.

Período Tairona

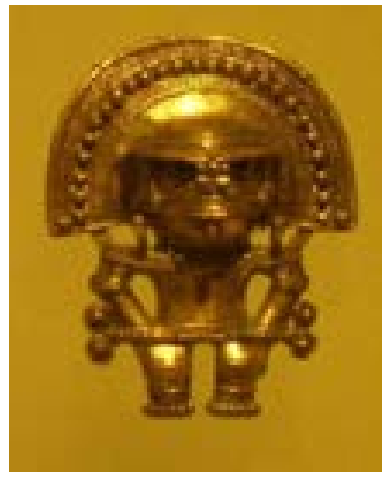

Hombre murciélago

\section{Propuesta; Hombre murciélago/ave}

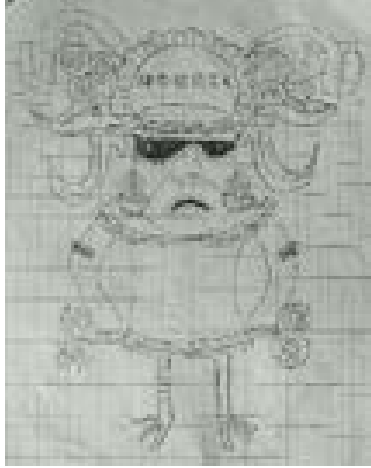

Diseño Icono cambio de regularidades
Icono orfebre.

Período Tairona

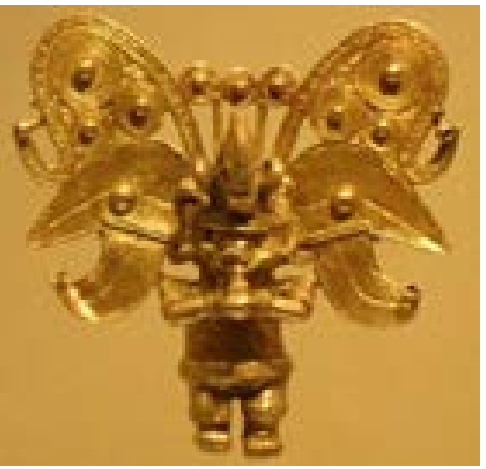

Hombre ave 
En especial después de la segunda visita al Museo del Oro de Bogotá, Colombia, entre las inquietudes que se presentan, se retoma la pregunta sobre cómo mantener la figura, 'al agrandarla' porque la mayoría de ellas son de tamaños "pequeños", y para especificar los detalles se hace necesaria una ampliación. Es de allí que se potencia la indagación hacia proporción y escalas.

\section{LAS ESCALAS - Razón y proporción}

La empresa común se encamina por el docente a partir de sugerir a los estudiantes que teniendo en cuenta la información acerca de simbología y representaciones de los taironas y/o muiscas, se interroguen si ¿podrán diseñar figuras (3D) similares? ¿Podrán crear figuras a escala teniendo en cuenta los patrones y regularidades encontrados en la clasificación?

Se manifiestan dificultades en la comprensión de aspectos proporcionales en una escala; el dibujo del objeto lo amplían o reducen, pero argumentan que la medida del objeto ha cambiado, así un cuadrado de longitud de su lado de 2 centímetros en escala 1:1, cuando es ampliado en el dibujo a la escala 2:1 o reducido a la escala1:2, para muchos estudiantes la medida del lado del objeto deja de ser 2 centímetros. También observa el profesor que dada la escala en la cual se encuentra un dibujo, a varios estudiantes se les dificulta argumentar si el dibujo es más grande o pequeño que el objeto real. Por lo tanto, el profesor propone revisar pausadamente las tareas y posteriormente escalas de exigencia cognitiva mayor, e invita a la realización de observaciones y examen de ellas. En esta temática el nivel de interés y de consulta de varios estudiantes es alto, sin embargo, para el uso de la información un gran número de ellos recurren al profesor o a los compañeros. La participación argumentada de los estudiantes disminuye y aumenta la responsabilidad que adjudican al profesor o a uno que otro estudiante.

En la culminación de este etapa el profesor no quiere dejar de lado la siguiente reflexión: "En las prácticas de identificación de regularidades y patrones también surgieron grupos pequeños, sin embargo sus miembros al igual que los grupos más numerosos, unos participaban activamente en discusiones y comentarios positivos, unos se encargaban de identificar el cómo y cuándo se debía tratar las actividades y otros aunque se hacían observaciones negativas nunca se dispusieron a la defensiva, entendiendo que debían asumirlo para crecimiento del grupo". A medida que las comunidades maduraron, algunos miembros del grupo toman buena parte del liderazgo. Cabe destacar que el profesor también evidencia miembros con participación periférica, miembros de la comunidad que en escasas ocasiones participaban y en otras oportunidades solamente lo hacían por una calificación. Pese a esto, algunos de estos miembros de participación periférica también mantienen el nexo con su grupo, guardando una actitud abierta y receptiva, para lograr que el grupo alcance su meta.

Las actividades han propiciado que los estudiantes inviertan tiempo fuera del aula para adelantar los proyectos donde ellos mismos gestionaron espacios de diálogo, esto es analizado por el profesor como un compromiso mutuo en mejora del aprendizaje.

Se evidenció un aprendizaje intergeneracional donde se notó agrupamiento libre; grupos que se buscaron por edades, por afinidades y perfiles. Hubo líderes que, cuando encontraron en sus grupos estudiantes buenos para el dibujo y otros que aún no habian reconocido su potencial y liderazgo. 
Así, la rúbrica general de niveles de participación en esta comunidad de práctica de clase, en cuanto a identificación y uso de patrones, cuando la empresa común toma diferentes matices entre razonamientos inductivos, la creación de figuras con nuevas regularidades y las tareas con escalas, presenta los siguientes datos (Tabla 7).

Tabla 7. Rúbrica general con niveles de participación de los estudiantes

\begin{tabular}{|c|c|c|c|}
\hline Categorias y niveles & 1 & 2 & 3 \\
\hline $\begin{array}{l}\text { Demanda de ayuda del } \\
\text { estudiante al profesor }\end{array}$ & $\begin{array}{l}\text { El grupo de estudiantes } \\
\text { necesita asesoramiento } \\
\text { continuado del profesor } \\
\text { para tomar decisiones }\end{array}$ & $\begin{array}{l}\text { El grupo de estudiantes } \\
\text { necesita asesoramiento } \\
\text { ocasional del profesor para } \\
\text { tomar decisiones propias }\end{array}$ & $\begin{array}{l}\text { El grupo de estudiantes } \\
\text { dialoga con el profesor y es } \\
\text { capaz de tomar decisiones } \\
\text { propias }\end{array}$ \\
\hline Raz. Inductivo & $2 \mathrm{D}-1 \mathrm{~B}$ & $4 \mathrm{E}$ & \\
\hline Creación & 1B & $4 \mathrm{E}$ & 2D \\
\hline Escalas & $1 B-2 D$ & $4 \mathrm{E}$ & \\
\hline $\begin{array}{l}\text { Utilización de recursos } \\
\text { para la actividad }\end{array}$ & $\begin{array}{l}\text { El grupo de estudiantes } \\
\text { usa únicamente los que } \\
\text { están disponibles }\end{array}$ & $\begin{array}{l}\text { El grupo de estudiantes busca } \\
\text { nuevos recursos a partir del } \\
\text { conocimiento de los que están } \\
\text { disponibles }\end{array}$ & $\begin{array}{l}\text { El grupo de estudiantes } \\
\text { busca y crea recursos de } \\
\text { forma genuina a partir } \\
\text { del conocimiento de los } \\
\text { disponibles }\end{array}$ \\
\hline Raz. Inductivo & 1B & $2 \mathrm{D}-4 \mathrm{E}$ & \\
\hline Creación & & $1 \mathrm{~B}-2 \mathrm{D}$ & 4E \\
\hline Escalas & 1B & $2 \mathrm{D}-4 \mathrm{E}$ & \\
\hline Formalidad & $\begin{array}{l}\text { El grupo de estudiantes no } \\
\text { fundamenta sus ideas y } \\
\text { delega su responsabilidad } \\
\text { en otros }\end{array}$ & $\begin{array}{l}\text { Solo en ocasiones el grupo } \\
\text { de estudiantes aportan ideas } \\
\text { fundamentadas y asume su } \\
\text { responsabilidad en la tarea }\end{array}$ & $\begin{array}{l}\text { El grupo de estudiantes } \\
\text { aportan ideas fundamentadas } \\
\text { y asume su responsabilidad } \\
\text { en la tarea }\end{array}$ \\
\hline Raz. Inductivo & 1B-2D & & 4E \\
\hline Creación & & 1B -2D & 4E \\
\hline Escalas & $1 \mathrm{~B}-2 \mathrm{D}$ & $4 \mathrm{E}$ & \\
\hline Relevancia & $\begin{array}{l}\text { Todas las ideas son poco } \\
\text { importantes o inadecuadas } \\
\text { para el objetivo marcado }\end{array}$ & $\begin{array}{l}\text { Algunas ideas son esenciales } \\
\text { o útiles para el del objetivo } \\
\text { marcado }\end{array}$ & $\begin{array}{l}\text { Todas las ideas son } \\
\text { esenciales o útiles para el del } \\
\text { objetivo marcado }\end{array}$ \\
\hline Raz. Inductivo & $1 B-2 D$ & $4 \mathrm{E}$ & \\
\hline Creación & & 1B- & $2 \mathrm{D}-4 \mathrm{E}$ \\
\hline Escalas & & $1 \mathrm{~B}-2 \mathrm{D}-4 \mathrm{E}$ & \\
\hline Autonomía & $\begin{array}{l}\text { El grupo de estudiantes } \\
\text { depende siempre de las } \\
\text { ideas y actitudes de los } \\
\text { compañeros o del profesor }\end{array}$ & $\begin{array}{l}\text { El grupo de estudiantes se } \\
\text { apoya en sus compañeros y } \\
\text { profesor para la realización de } \\
\text { tareas y exposición de ideas }\end{array}$ & $\begin{array}{l}\text { El grupo de estudiantes } \\
\text { toma la iniciativa para } \\
\text { la realización de tareas, } \\
\text { exposición de ideas }\end{array}$ \\
\hline Raz. Inductivo & & $1 B-2 D$ & 4E \\
\hline Creación & & $1 \mathrm{~B}-2 \mathrm{D}-4 \mathrm{E}$ & \\
\hline Escalas & & $1 \mathrm{~B}-2 \mathrm{D}-4 \mathrm{E}$ & \\
\hline Originalidad & $\begin{array}{l}\text { Las ideas están copiadas } \\
\text { de las fuentes disponibles. } \\
\text { Sin embargo, contienen } \\
\text { errores de conexión. }\end{array}$ & $\begin{array}{l}\text { Las ideas están parafraseadas } \\
\text { de las fuentes disponibles. Sin } \\
\text { embargo, contienen errores de } \\
\text { conexión. }\end{array}$ & $\begin{array}{l}\text { Las ideas están construidas } \\
\text { a partir de la lectura de varias } \\
\text { fuentes y expresadas de } \\
\text { forma creativa. }\end{array}$ \\
\hline Raz. Inductivo & 2D & 1B & $4 \mathrm{E}$ \\
\hline Creación & 2D & 1B & $4 \mathrm{E}$ \\
\hline Escalas & 1B & $2 \mathrm{D}-4 \mathrm{E}$ & \\
\hline
\end{tabular}


Atendiendo a los parámetros establecidos según los cuales se considera Participación periférica legítima si la valoración media de los aspectos se encuentra en el intervalo [1,2); Participación legítima si la valoración media de los aspectos se encuentra en el intervalo $[2,3)$ y Participación plena si la valoración media de los aspectos es 3. En esta comunidad de clase se ha presentado mayoritariamente participación periférica legítima y la participación legítima se manifiesta principalmente en uno de los grupos que formó parte del grupo de estudiantes.

\section{Sesión 4. Consolidación de la participación}

En un salón especializado para exposiciones de arte que facilitó la administración, este permitió integrar de manera amplia y ordenada los proyectos y su socialización, no solo a sus pares, sino también invitados docentes del grupo de profesores de la Facultad en la cual se desarrolla la innovación, sino también a personas externas a la universidad y visitantes académicos que en ese momento se encontraban en un congreso internacional de la historia de la matemática.

Cabe resaltar que la población que integran las comunidades son estudiantes de bajos recursos, que de principio no conocían este salón de exposiciones y que le dio una importancia mucho mayor al evento, además, hay un ambiente institucional de cierre de semestre y es la hora de demostrar lo aprendido, tanto, el profesor como sus estudiantes.

Figura 6. Creaciones de los estudiantes en sala de exhibición - Creaciones propias.

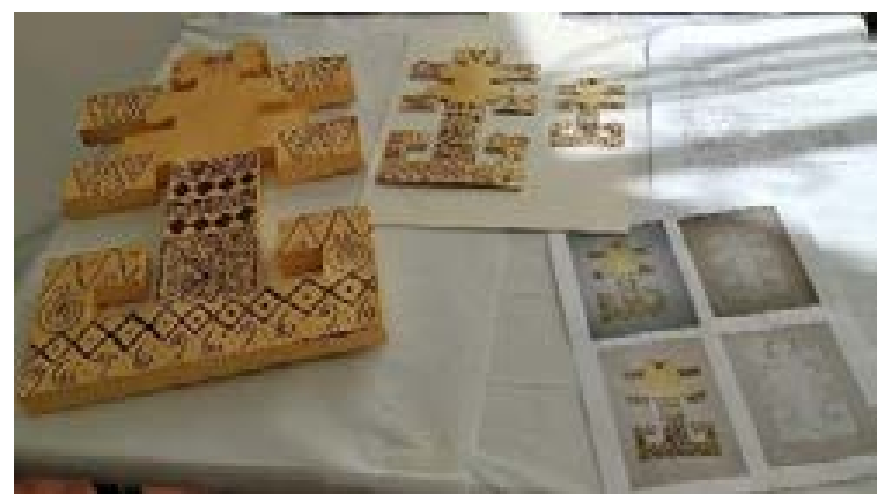

En este montaje de exposición, las creaciones de figuras del arte precolombino a diversas escalas y su explicación estuvieron a cargo de los grupos de estudiantes (Ver figura 6).

Figura 7. Socialización de algunos proyectos - Creaciones propias.

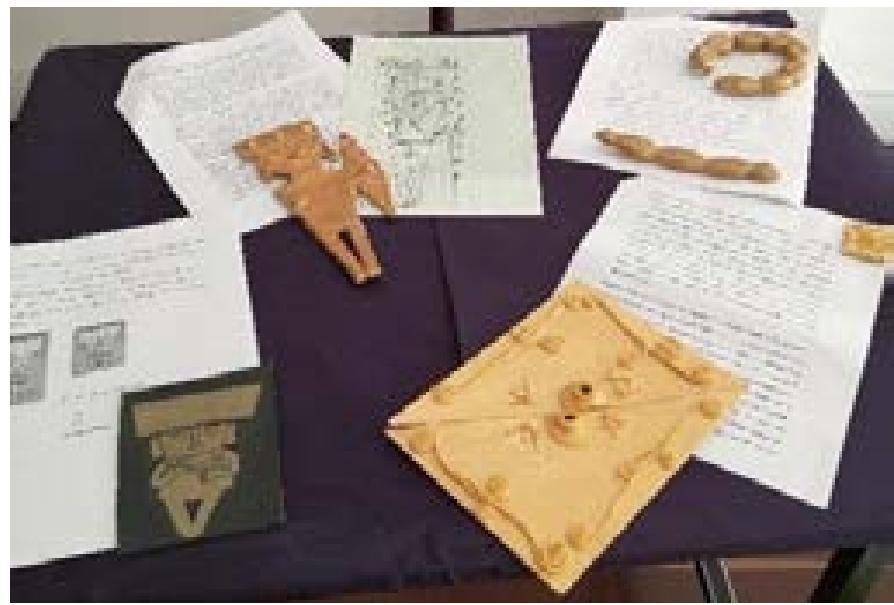


La socialización de las creaciones en 3D a partir de la argumentación que les brinda el estudio de las culturas precolombinas, se realiza en un ambiente de compromiso y dedicación, buscando que todo impacte en la autoestima de los miembros de las comunidades. Aquí el profesor contó con apoyo de la maestra de artes plásticas y visuales y la investigadora en educación matemática - compañera de área-, tanto en el montaje de la exposición artística como en los demás pasos de la organización se buscó integrar a los estudiantes, como aprendices, para gestionar todo lo correspondiente a esta exposición de producciones, en un cierre, donde se integra las comunidades en un nuevo espacio (Ver figura 7).

\section{CONCLUSIONES}

Al observar y analizar las figuras precolombinas, nos expresamos en función de elementos característicos, por ser la terminología que surge al explicar, en la parte formal, lo hallado en las figuras para la investigación. La investigadora en el área de educación matemática observó que esta era una expresión apropiada para un primer acercamiento al tema de regularidades y patrones. El vocabulario y terminología específica se asumen dentro de la investigación para hablar de los objetos precolombinos con una mirada de reconocimiento y análisis formal.

El material visual, como parte complementaria e imprescindible de esta propuesta, surge en las fases iniciales del desarrollo de la investigación con la elaboración de un primervídeo examinando la cultura y zona arqueológica Muisca. Esto nos habla del trabajo juicioso de pensar la imagen como elemento clave en la propuesta de análisis de regularidades y patrones y específicamente centrar la atención en la figura precolombina imprimiendo valor al entendimiento inmerso en estos objetos, así como en la oportunidad de traer a conocimiento de los estudiantes una parte de la historia y de la cultura.

La acción de agente de cambio se potenciará si existen condiciones adecuadas, algunas de ellas hacen referencia a: una sociedad que aprende a valorar una herencia cultural desde el estudio y reconocimiento, entendiéndose e integrándose como un factor positivo, la existencia de mecanismos dirigidos a la implicación de las personas (comunicación positiva y constante, motivación, espacios de participación y atención a la ansiedad) y una flexibilidad que permita acciones para vencer las resistencias (acompañamiento, formación, asesoramiento y recursos).

En estos procesos de innovación, se rescata la importancia de una participación abierta que intenta enfocarse en una estructura horizontal, que motiva al profesor a adaptarse a una realidad cambiante y compleja y se vincula a prácticas muy concretas con pares, de creación y participación, a las que tiene que atender.

Si bien inicialmente las actividades se encaminan hacia análisis muy finos concernientes a patrones de repetición, esta observación de regularidades y patrones en orfebrería precolombina coadyuvó al desarrollo de razonamientos inductivos y posibilita construcciones mentales en el proceso de generalización y abstracción; en las cuales el profesor espera se apoye el estudio de otros conceptos matemáticos.

El ejercicio de aprendizaje desde la perspectiva de las comunidades de práctica genera y desarrolla en el profesor competencias de indagación, innovación y seguimiento, durante un proceso de formación, que le exigen articular más efectivamente la actividad académica con el entorno, cumpliendo las funciones sustantivas de docencia, investigación y difusión de la cultura. 
En esta comunidad de clase se ha presentado mayoritariamente participación periférica legítima y la participación legítima se manifiesta esporádicamente. Concerniente a la participación plena se plantea que el continuar con este estilo de trabajo en los cursos de análisis matemático puede aportar en la práctica discursiva.

El seguimiento a la participación de los estudiantes permite establecer avances en cuanto a la construcción de razonamientos inductivos, la toma de decisiones a partir de la indagación con mayor independencia con respecto al profesor y un compromiso en la creación de las figuras e informes de las tareas del grupo.

Atendiendo a la participación de los estudiantes y sus conocimientos concernientes a la identificación de regularidades y patrones, queda abierta una pregunta sobre la enseñanza transversal de estas prácticas matemáticas, en especial en cuanto al uso de regularidades y patrones en la enseñanza desde la primera infancia hasta los diversos ciclos de educación superior.

Reconocimiento a los tiempos asignados para esta indagación mediante resoluciones 893, 051 y 764 de 2021 concerniente al apoyo al Centro de Educación Matemática de la Universidad Colegio Mayor de Cundinamarca, tiempos que fueron útiles para las revisiones de esta publicación. Un agradecimiento a la investigadora Nury Vargas Hernández del grupo BIOMA por su participación con tiempos propios, destinados a los momentos de escritura.

\section{REFERENCIAS BIBLIOGRÁFICAS}

Blanco, H. (2006). La Etnomatemática en Colombia. Un programa en construcción. (M. Borba, Ed.) Revista BOLEMA: Boletim de Educação Matemática, 19 (26), 49-75.

Camargo, L. (2010). Descripción y análisis de un caso de enseñanza y aprendizaje de la demostración en una comunidad de práctica de futuros profesores de matemáticas de educación secundaria. Disertación doctoral. España: Universidad de Valencia.

Carrillo, J., Climent, N., Gorgorió, N., Rojas, F. \& Prat, M. (2008). Análisis de secuencias de aprendizaje matemático desde la perspectiva de la gestión de la participación. Enseñanza de las Ciencias, 26(1), 67-76.

Franke, M. L., Kazemi, E. y Battey, D. (2007). Understanding teaching and classroom practice in mathematics. En F. Lester (Ed.), Second handbook of research on mathematics teaching and learning (pp. 225-256). Charlotte, USA: IAP-NCTM.

Gairín, J. (2015). Las comunidades de práctica profesional: creación, desarrollo y evaluación. Las Rosas, Madrid. Wolters Kluwer. España, S.A.

Lave, J., y Wenger, E. (1991). Situated learning. Legitimate peripheral participation. Cambridge: University Press.

Legast, A. (1987). El animal en el mundo mítico Tairona. Fundación de Investigaciones Arqueológicas Nacionales Banco de la República. Bogotá, Colombia Read, H.(1990). Arte y Sociedad. Ediciones Península, Madrid. 
Morales, R., Cañadas, M. C. y Castro, E. (2017). Generación y continuación de patrones por dos alumnas de 6-7 años en tareas de seriaciones. PNA, 11(4), 233-252.

Read, H. (1990). Arte y Sociedad. Ediciones Península, Madrid.

Sondereguer, C. (2000). Diseño precolombino. Catálogo de iconografía: Mesoamérica,

Centroamérica, Suramérica. Ediciones G. Gili, S.A: México.

Sondereguer, C. (2003). Manual de iconografía precolombina y su análisis morfológico. Marco teórico Cátedra Sondereguer de Diseño y Arte Precolombino. Facultad de Arquitectura, Diseño y Urbanismo. Universidad de Buenos Aires. Recuperado en:

https://www.academia.edu/26967799/ Sondereguer_Cesar_Manual_de_ iconografia_precolombina_y_su_ analisis_morfologico_pdf

Reichel-Dolmatoff, G. (2005). Orfebrería y Chamanismo. Un estudio iconográfico del Museo del Oro del Banco de la República de Colombia. Villegas Editores: Colombia.

Rojas, C. (2013) La Etnomatemática: un campo fértil para la investigación. Revista Ejes, 1(1), pp. 10-13.

Steen, L. A. (1988). The Science of Patterns. Science, 240 (4852), 611-616.

Vargas, N y Vargas, J. "Arte y matemáticas. El caso de regularidades y patrones en la cultura precolombina para la enseñanza en precálculo". En: Champaign, Illinoi, EE. UU. por Common Ground Research Neworks, NFP. P. 58, 2019

Vargas, J., Vargas, N. y Cáceres, M. J. (2020). El profesor y el arte precolombino: creación e interdisciplinariedad en las prácticas matemáticas de identificación de regularidades y patrones. Revista Boletín Redipe, 9(12), 320-334. https:// doi.org/10.36260/rbr.v9i12.1158

Wenger, E. (1998). Comminities of practice. Learning, meaming and identity. Cambridge, Cambridge University. 